\title{
Personalized Recommendation for Individual Users Based on the Group Recommendation Principles
}

\author{
Michal KOMPAN, Mária BIELIKOVÁ \\ Institute of Informatics and Software Engineering, \\ Faculty of Informatics and Information Technologies, \\ Slovak University of Technology in Bratislava, \\ Ilkovičova, Bratislava, 842 16, Slovakia, \\ \{kompan, bielik\}@fiit.stuba.sk
}

\begin{abstract}
The amount of information available on the Web is increasing day by day. Users are overloaded and cannot access desired information in a reasonable time. Plenty of approaches for the web personalization, which try to solve information overload have been proposed in the literature. Important feature of any personalization is its accuracy, or more precisely accuracy of personalized recommendation provided to a user. In this paper we propose a new recommendation approach for the single-user collaborative filtering based on the principles of the recommendations for group of users. We explore the best configuration for such an approach according to the group size used for computation, the aggregation strategy of ratings used within groups or the number of similar users used for the recommendation. We did an experiment over the MovieLens and SME.SK news portal datasets. Proposed approach is compared to the standard collaborative and group recommender respectively. The results support our hypothesis that the proposed approach brings statistically significant improvement and it is applicable on various domains, thus can be used for the single-user recommendation tasks.
\end{abstract}

Keywords: Collaborative filtering, group recommendation, virtual groups, aggregation strategies.

\section{Introduction and Related Work}

Recommender systems are an integral part of the Web nowadays. The need for the personalized web increases day by day, while people are generally overwhelmed by the amount of information available over the Web. Personalized web enables users to get access to relevant information matching their interests by filtering or recommending interesting items. Personalized recommendation becomes crucial for the business sector, where it can help to increase profits (e.g., recommending interesting products increases the chances of getting a purchase orderalso by increasing users' visits of the web sites). Personalized recommendation is the most used approach to satisfy both-users and the business sector respectively.

The recommendation task can be defined as:

$\forall u \in U, s_{u}^{\prime}=\arg \max _{s \in S} f(u, s)$

where $U$ represents users, $S$ represents the recommendation objects and $f$ is the usefulness function (usefulness of the objects for specific user $u$ ).

Several approaches have been proposed for the personalized recommendation. Two principal approaches include content-based and collaborative recommendation. In order to obtain better results these two approaches, are often combined in so-called "hybrid" approaches [5].
The content-based recommendation uses the similarity between recommended items (user liked an article about a new car, thus he/she will probably like a similar article about cars). The similarity between recommended items and a user profile can be computed based on numerous aspects such as a simple text similarity or advanced content analysis is performed. Moreover, various enhancements for specific domains as news have been proposed [11, 24, 25].

The second approach is collaborative recommendation (filtering). This approach to personalized recommendation instead of the content similarity takes advantage of user to user similarity, which is usually computed based on user's content ratings (e.g., the reader $A$ likes articles about cars and politics, the reader $B$ likes articles about cars, thus the reader $B$ will probably like also articles about politics similarly to the reader $A$ ).

Three basic models to collaborative filtering can be distinguished in respect to the focus of the computation process: user-based, modelbased and item-based approaches [6]. The userbased approach creates sets of user neighbors (similar users) and then the assumption that similar users like similar items is applied. On the contrary, the item-based approach creates set of similar items, while the rating is computed based on the similar items user ratings. Finally, the model-based approach 
constructs users sets while the rating is derived based on the other users (within the set) ratings.

Various approaches within the collaborative recommendation have been proposed. The matrix factorization models as SVD, SVD++, PLSA or neural networks are comparable to the state-of-art approaches, while they often offer memory efficient model [13]. Neighborhood based models are used more often, thanks to their simplicity and possibility to easily understand the reason for providing specific recommendations (as this is one of the recommender system important characteristics). Providing explanations for the recommendation is often crucial from the user's satisfaction point of view.

In order to obtain best results, these approaches are often mixed. For example, in order to create scalable news recommendation Das et al. proposed recommender system based on PLSI and MinHash as one of model-based approaches and item co-visitation as a representative of the user-based approach [8].

From the other point of view we distinguish a single-user and group recommenders. In the last years the phenomenon of social networking [18] and mobile devices increase has brought us to the increasing demand for recommendations designed for groups of users [12], because of the group oriented domains increase (e.g., TV, movie, holidays). The possibility of the usage of group recommendation approaches within standard single-user recommendation was raised by Masthoff [14]. In the group recommendation we use not only content or users' similarity, but inter-group relations are considered (derived explicitly or implicitly [10]) in order to provide recommendation for the whole group instead of single-user.

Most of the proposed group recommendation approaches deal with TV or music domains as these include activities which are usually performed in groups of users [4]. The classic example of such a system is MusicFX [16], which was designed to influence a music played in the gym by actual present users. Ntousi et al. proposed the framework gRecs [19], which uses agglomerative hierarchical clustering in order to compute and predict user's and thus group's preferences. Recently the group recommendation was used in new domains as holiday or restaurant recommendation [17, 2].
In the group recommendation members of the group do not include only similar users (groups are formed naturally such as people in the cinema, bus, watching TV etc.), so some kind of aggregation of single-user recommendations or single-user preferences has to be performed in order to produce one list of recommendation for every group member.

As the group recommendation process is highly dependent on the aggregation strategy used, several strategies have been proposed [15]. When the standard plurality voting is used, several users can be highly unsatisfied (the minority is outvoted). Strategies, when the majority of users take into account (e.g., average, dictatorship) are generally considered as strategies without minimal satisfaction (the minimal level of satisfaction of every group member is not guaranteed). On the contrary, strategies as least misery or fairness ensure that the minimal satisfaction for every item and user is guaranteed across the group members.

Broadly speaking, while the standard singleuser recommendation tries to satisfy actual user needs, the group recommendation based on the used aggregation strategy and the goal of the recommendation tries to maximize satisfaction of every user of the group.

In this paper we propose a novel approach for the collaborative recommendation. We explore the potential of usage group recommendation principles to generate recommendations for a single-user. We believe that recommendations based on group principles can introduce the recommended items variety. Moreover, based on used aggregation strategy, proposed approach can be applied in various settings and domains and thus help us to overcome some standard collaborative recommender shortcomings as the cold-start problem. We investigate the influence of several aspects as the group size or number of users used for the recommendation to the proposed approach effectiveness.

This paper has two primary contributions:

Exploring the usage group recommendation principles in the individual personalized recommendation by proposing new domain independent recommendation approach.

Analysis of aggregation strategies used for the group preference aggregation and number of similar users used for the collaborative filtering. 
The paper is organized as follows. Section 2 describes the proposed approach in three basic steps: virtual groups construction, similarity computation and recommendation. In Section 3 we describe experiments focused to reveal performance aspect of each step of proposed approach. We conclude the paper with discussion on method properties based on performed experiments and outline future work directions.

\section{Group Principle to Single-user Recommendation}

We propose the recommendation method which extends the task of the group recommendation described above to the single-user recommendation. The aggregation of singleuser profiles in order to obtain one group profile combines users' preferences and also in some settings can introduce variety (in the mean of users diversity), which can be beneficial for the recommendation.

The main difference between classic collaborative recommendation and our proposed approach (see Figure 1) is that we generate recommendation not based on the user to user similarity, but based on the similarity between the user and virtual users.
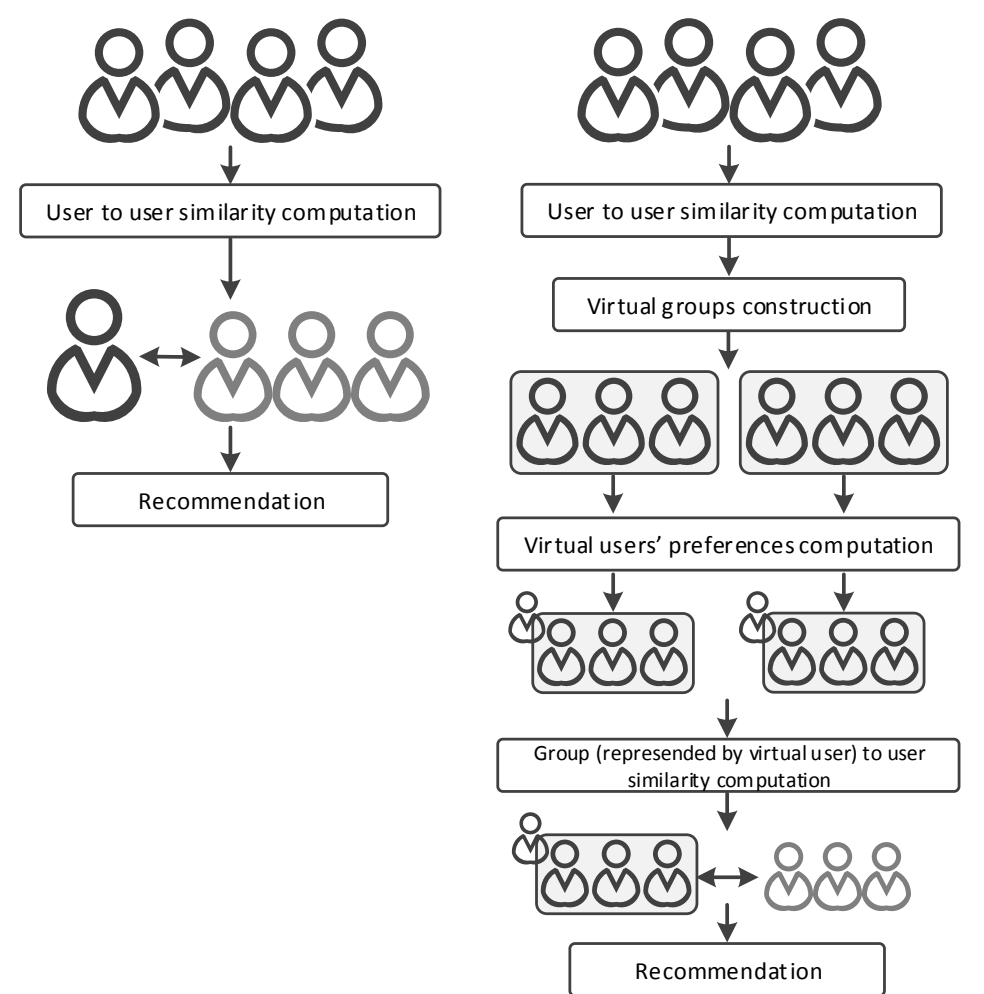

Figure 1. Standard collaborative recommendation approach (left) compared to the proposed single-user group approach (right). The gray box represents a virtual group.

Virtual group is pseudo randomly generated group from available users in the system. These users do not know each other and there is no factual relationship between these users (i.e., virtual group does not consider social relationships present in real life).

Virtual user is a member of the virtual group. It is created as the preferences aggregation of the virtual group members. Various aggregation strategies for computing single-user preferences can be used, e.g., average strategy computes the average rating of item across the group members and this average rating is assigned to the virtual user.

Every user is assigned just into one virtual group, while the virtual group preferences are represented by the virtual user (based on the aggregation strategy used). While some archers use the aggregation strategy to fill unknown user's preferences [1], we use the aggregations in order to create virtual users (which represent the virtual group preferences).

Proposed approach differs from standard clustering approaches, while we do not consider the group of users as a group of similar users. Moreover, every user in the virtual group gets own personalized list of recommendations, which usually differs from other group members. Thanks to various 
settings as group size or inner-group similarity, it is possible to control and improve results in order to fulfill specific goal - to obtain various results or to focus on the specific interest area.

Our approach (see Algorithm 1) consists of three basic steps:

1. virtual groups construction (Algorithm 1, lines 1-4);

2. similarity computation between virtual users and real users outside the group (Algorithm 1, lines 5-8);

3. generation of recommendation for specific user (Algorithm 1, lines 9-11).

Algorithm 1. Proposed collaborative recommender approach based on group recommendation.

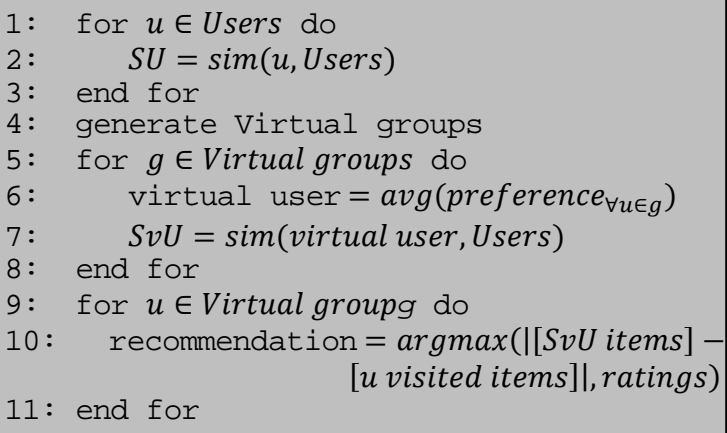

\section{Virtual group construction}

Every user is assigned to one virtual group, which preferences are represented by a virtual user. These groups are generated pseudorandomly based on the inter-group similarity, which is computed as the average of standard cosine real user to real user similarities. Next, the preferences of a virtual user are computed. For this purpose we use an aggregation strategy adopted from the group recommendation.

Primarily, we construct virtual user's preferences by using average aggregation strategy, which takes users' preferences (ratings) and calculates the average rating of specific item for the whole group. Various aggregation strategies may be used with or without minimal satisfaction guarantee. From the point of view that such result of aggregation is in the next step used for similarity computation, strategies with minimal satisfaction should bring better results (predicted rating accuracy). In this manner we obtain preferences of a virtual user, which represent (aggregated) preferences of the whole group.

Moreover, the real-life groups derived from the social networks or the other web activity may be used as the baseline for the group construction. Likewise, various group sizes can be constructed, while it is clear that larger groups should increase the variety of recommended items and vice versa. Moreover, users with various activity levels can be mixed in order to bring more recent results when the domain of recommendation is highly dynamic. Standard group sizes used by researches during past experiments with the group recommendation involve 3-7 members [21].

\section{Similarity computation}

Similarity is in this step computed between created virtual groups, which preferences are represented by virtual users and other real users outside the groups. Next, the most similar users (to the virtual group represented by virtual user) search is performed. This is similar to the standard single-user collaborative recommendation except that not the user to user similarity is computed, but the user to the virtual group (virtual user) similarity is computed. For the task of the similarity computation, we involve standard and widely used cosine similarity. In this manner we obtain a list of the most similar users for specific group based on the average ratings of content within the group members (represented by the virtual user).

\section{Recommendation}

The final step consists of generating recommendation for the specific user, whose preferences are represented as the average of the group (by the virtual user). The recommendation approach is similar as the Top-N collaborative approach. When recommending to the specific user of the virtual group, we look for the most visited and highly rated items by the similar users (similar real users outside the group) based on the virtual to real users similarity computation (step 2), which were not visited by the user to whom the recommendation is generated. These items are reordered based on the number of visits and highest rating obtained and Top-N items are recommended.

In this manner we obtain a list of recommendations for every user in the group. The group members do not obtain same recommendations, but every group member receives its own personalized list. It is clear that various approaches to the group construction may lead to various results in the context of 
recommendation precision or from the recommendation goal point of view (e.g., learning process maximisation).

The groups used for the recommendation can be created by several approaches. We use random group construction, while the innergroup similarity is considered. In the real life users belongs to various groups - natural or virtual. These groups can be used similarly as virtual groups, we generated, but the influence for the recommendation needs to be investigated. It is clear that some kinds of groups tend to consist of users with similar preferences, but some do not. In the other hand, as we discovered in our experiments, groups with high inner-similarity bring not so good results as the average similar groups.

\section{Evaluation}

To investigate the influence of specific parameters as the group size, the inner-group similarity or the similar user neighbourhood, we developed single-user recommender system, which is based on the proposed new recommendation approach. Similarly, we developed standard single-user collaborative recommender (Figure 1 left), in order to compare expected improvements. The standard Top- $\mathrm{N}$ collaborative approach was designed as follows - Algorithm 2.

Algorithm 2. Standard Top-N collaborative filtering approach.

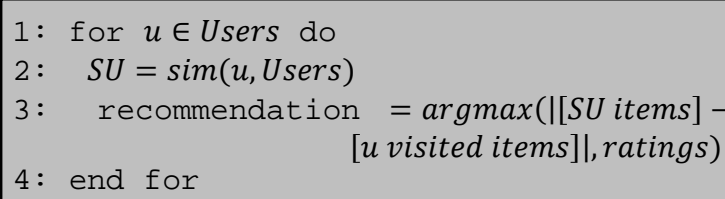

For the experiments we use the MovieLens (http://www.grouplens.org) 100k dataset, which is widely used as the gold standard dataset for recommender systems' evaluation. The dataset consists of 100.000 ratings (scale 1-5) from 943 users on 1682 items (minimal 20 ratings per user). The dataset was split into train (80\%) and test data (20\%). In addition, 5 fold cross validation was performed.

We involved several metrics widely used for recommender system's evaluation. The Precision@3 and Precision@10 are computed as standard Precision metrics for the top 3 and top 10 recommended items respectively. Correspondingly, we computed the Mean
Absolute Error (MAE (2)) and the Root Mean Absolute Error (RMSE (3)) in order to measure predicted ratings [22]. While the RMSE prefers more and small errors, the MAE prefers larger and few errors.

$$
\begin{aligned}
& \text { MAE }=\sqrt{\frac{1}{|\tau|} \sum_{\mathrm{u}, \mathrm{i} \in \tau}\left|\mathrm{r}_{\mathrm{ui}}^{\prime}-\mathrm{r}_{\mathrm{ui}}\right|} \\
& R M S E=\sqrt{\frac{1}{|\tau|} \sum_{\mathrm{u}, \mathrm{i} \in \tau}\left(\mathrm{r}_{\mathrm{ui}}^{\prime}-\mathrm{r}_{\mathrm{ui}}\right)^{2}}
\end{aligned}
$$

Users in the dataset rated the items on the 5 levels scale. Thus the rating of level 3 can be considered as the average, while levels 1 and 2 refer more to the negative feedback. Because of this, in our experiments were used for the recommendation only those items, which ratings are above and including 3 . In order to provide objective comparison, the results provided in experiments are average results when the positive rating is considered as rating 4 and 5 and on the other side when positive rating is considered as 3, 4 and 5 .

Second dataset used for the evaluation is SME.SK (http://www.sme.sk) which refers to the standard news portal domain. The dataset subset used in experiments consists of 1200 users and their activity (list of visited articles average 14 on user). The dataset is characteristic with small user activity (cookie identification) compared to the number of article possible to read. For this reason the recommendation was not evaluated to the specific article ID - visited during the test period, but the combination of section and category (assigned to every article-total combination possible is 420) was used. Similarly, the dataset was spilt into train and test subsets (80\% and $20 \%$ ).

The experiments were performed in order to answer several questions. As the virtual group construction is the critical and most important step in proposed approach, we focus on the group characteristics as the group size, intergroup similarity and the approach for virtual user's (aggregation strategy) preferences generation. Moreover, the number of similar users used for the recommendation was investigated. In addition we compared results of proposed approach on datasets from different domains, in order to study the domain dependency of proposed approach. 


\section{Results}

Numerous collaborative approaches for Top- $\mathrm{N}$ recommendation have been compared based on the MovieLens dataset [7,23]. The results show that the precision $(N=1 . .20)$ does not exceed $40 \%$ [26]. When comparing algorithms based on MAE [9], the average difference - predicted and true rating is approx. 0.5 for the today's approaches.

In our experiments we firstly focus on the user and group similarity. The virtual groups were generated pseudo-randomly. For every group size (3-7 members) we generated groups with various inner-groups similarities (0.0-0.5) based on the standard cosine computation. The inner-group similarity was computed as the average of the user to user similarity (based on the rating history). Thus we obtained 190 groups for every group size which brings us in total 950virtual groups of various size and inner-group similarities. virtual group members have to be aggregated into one virtual user's preference. In the group recommendation several aggregation strategies have been proposed. In our experiments (Table 1) we compared standard Average strategy (average of single user ratings), Least Misery strategy (minimum of ratings is used) and the Most Pleasure strategy (maximum of ratings is used).

As we can expect, the Average strategy performed the best from the precision point of view, while the majority of users can bias the aggregated rating. When the MAE or RMSE is used for the comparison, Least Misery followed by Most Pleasure strategy obtains best results. The result suggests that in the virtual groups, in the most occurrences, some of the users deviate from the rest of the group in the mean of the higher ratings. This is supported by the inter-group similarities and the result of Average strategy. Because of

Table 1. The aggregation strategies comparison.

The Average (AV), the Least Misery (LM) and the Most Pleasure strategy (MP).

\begin{tabular}{|c|c|c|c|c|c|c|c|c|c|}
\cline { 2 - 11 } \multicolumn{1}{c|}{} & \multicolumn{3}{c|}{ P@3 } & \multicolumn{3}{c|}{ MAE } & \multicolumn{3}{c|}{ RMSE } \\
\hline Group size & AV & LM & MP & AV & LM & MP & AV & LM & MP \\
\hline 3 & 0.5733 & 0.5250 & 0.5711 & 0.60 & 0.53 & 0.58 & 0.49 & 0.40 & 0.48 \\
\hline 4 & 0.5750 & 0.5739 & 0.5575 & 0.60 & 0.60 & 0.59 & 0.50 & 0.50 & 0.49 \\
\hline 5 & 0.5616 & 0.5592 & 0.5588 & 0.61 & 0.60 & 0.60 & 0.50 & 0.50 & 0.50 \\
\hline 6 & 0.5555 & 0.5538 & 0.5541 & 0.57 & 0.57 & 0.57 & 0.47 & 0.47 & 0.47 \\
\hline 7 & 0.5397 & 0.5346 & 0.5356 & 0.56 & 0.55 & 0.56 & 0.47 & 0.46 & 0.46 \\
\hline Avg. & $\mathbf{0 . 5 6 1 0}$ & 0.5493 & 0.5554 & 0.59 & $\mathbf{0 . 5 7}$ & 0.58 & 0.49 & $\mathbf{0 . 4 7}$ & 0.48 \\
\hline
\end{tabular}

When comparing various group similarities, from the precision point of view, an interesting fact was discovered. The best performers were not the most similar groups (inner-similarity), but the groups with inner-similarity between 0.2 and 0.4 . This can be explained by the variety, when more interesting movies or articles were introduced into the group profile, while some member similarity was still guaranteed. Thus, in further experiments we use groups of such inner-similarity values.

In order to calculate the group to user similarity, which is needed for the proposed recommendation approach, the preferences of small differences, the Average strategy is used for the further experiments.

Next we experimented with the group size and with the number of similar users used for the recommendation. As the both Top- $\mathrm{N}$ and proposed new collaborative approach respectively uses the similar users to predict the user's interest (ratings), we created recommendations based on several user sets (1101 most similar users). The results clearly show that the standard collaborative recommender brings the best results based on 41 similar users' set (Figure 2). In the opposite, our proposed recommender based on the group 


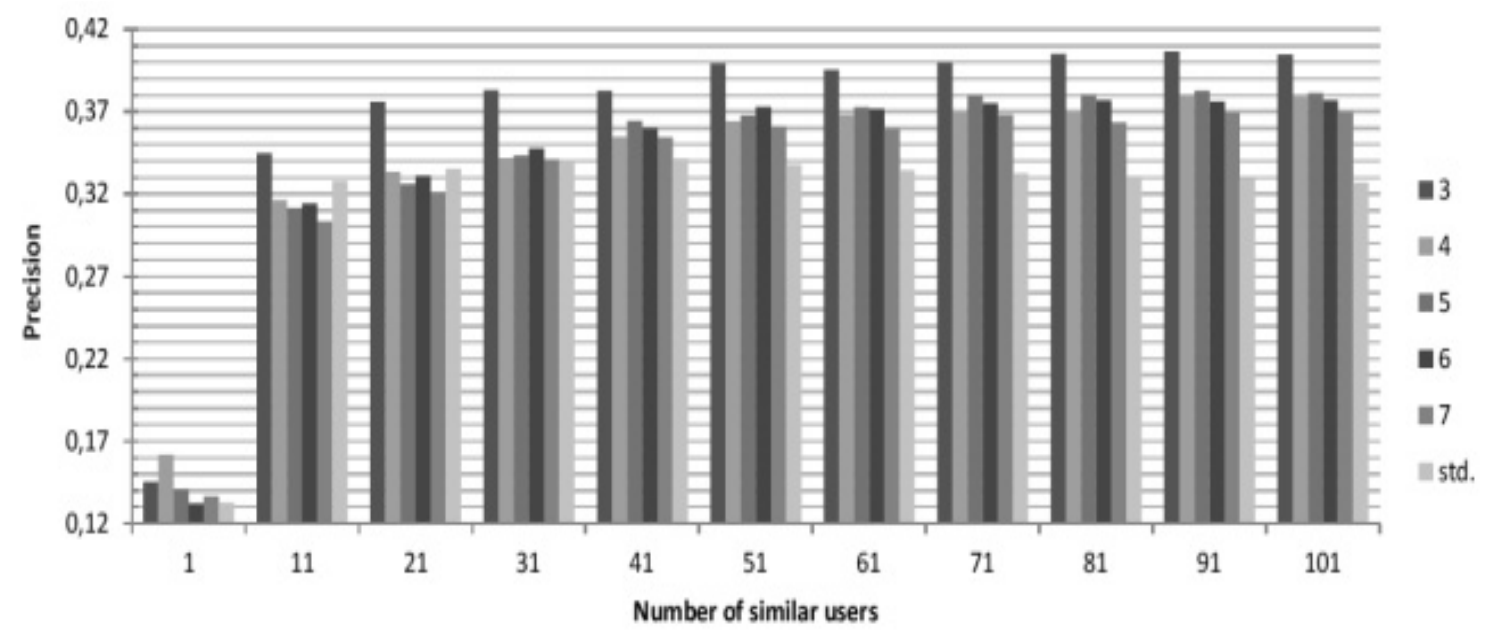

Figure 2. Precision@3 for various numbers of similar users used for the recommendation (1-101) and various group sizes (3-7). Results of standard collaborative recommender are also presented (std.)

- MovieLens dataset -

Table 2. Impact of the similar user set size (Top N user used for recommendation) on the proposed approach and the standard Top-N (std.) recommendation. Values represent P@3.

\begin{tabular}{|c|c|c|c|c|c|c|c|}
\hline \multirow{2}{*}{$\begin{array}{l}\text { Similar user } \\
\text { neighborhood }\end{array}$} & \multicolumn{5}{|c|}{ Group size } & \multirow[b]{2}{*}{ Avg. } & \multirow[b]{2}{*}{ Std. } \\
\hline & 3 & 4 & 5 & 6 & 7 & & \\
\hline 1 & 0.1453 & 0.1618 & 0.1408 & 0.1322 & 0.1367 & 0.1434 & 0.1327 \\
\hline 11 & 0.3447 & 0.3165 & 0.3113 & 0.3144 & 0.3035 & 0.3181 & 0.3279 \\
\hline 21 & 0.3760 & 0.3335 & 0.3261 & 0.3311 & 0.3210 & 0.3375 & 0.3353 \\
\hline 31 & 0.3830 & 0.3418 & 0.3433 & 0.3478 & 0.3410 & 0.3514 & 0.3405 \\
\hline 41 & 0.3827 & 0.3547 & 0.3645 & 0.3600 & 0.3546 & 0.3633 & 0.3415 \\
\hline 51 & 0.3991 & 0.3642 & 0.3677 & 0.3729 & 0.3612 & 0.3730 & 0.3377 \\
\hline 61 & 0.3954 & 0.3681 & 0.3727 & 0.3717 & 0.3598 & 0.3736 & 0.3344 \\
\hline 71 & 0.3997 & 0.3695 & 0.3799 & 0.3749 & 0.3682 & 0.3784 & 0.3326 \\
\hline 81 & 0.4051 & 0.3697 & 0.3801 & 0.3771 & 0.3635 & 0.3791 & 0.3310 \\
\hline 91 & 0.4065 & 0.3797 & 0.3827 & 0.3764 & 0.3696 & 0.3830 & 0.3289 \\
\hline 101 & 0.4046 & 0.3791 & 0.3813 & 0.3769 & 0.3699 & 0.3824 & 0.3271 \\
\hline
\end{tabular}

recommendation approaches brings better results when the size of similar users' set is between 91 and 101 (Table 2). Because interests of a single user are more clearly expressed when standard recommendation is used, the decreasing trend over the similar user set size of precision can be observed. In the contrary, the opposite can be observed when our approach is used, while the virtual preferences partially "blur" the specific user's preferences.

Generating virtual groups allows us to group users with similar behaviour pattern (e.g., sporadic visit) together. Moreover, more than one group can be assigned to the user - to the inactive users' group can be added highly 
active user in order to introduce the variety and topicality of recommended items.

A paired t-test was performed to determine if the proposed single-user recommendation based on the group recommender principles is effective. The difference is considered to be statistically significant $(p=0.0018$, $\alpha=0.05, t=4.21$ ), thus proposed approach brings better results as the standard Top-N collaborative recommender.

While the precision of proposed approach is generally decreasing with the size of the group used for recommendation, MAE and RMSE is improving with the group size (best performers group size 7 , Top $3-0.48$ and 0.40 ; Top 10 0.58 and 0.49). This is an expected result. Based on the assumption that when more users is used for the rating prediction, their preferences are closer (more accurate) to the ideal "rating" (single-user variances are eliminated). From the other hand, the difference between predicted ratings over various group size is very small and in the average it is almost identical to the standard collaborative approach. When compared the best performer (group size 3 and 91 similar users) to the standard approach (group size 3), our proposed approach brings the improvement more than $7.8 \%$ (11.5\% when ratings 3,4 and 5 used) for the P@3 and 6.7\% (10.4\% when ratings 3,4 and 5 used) for the P@10 respectively. This is a huge improvement for the recommender approach and thus indicates that proposed approach can be used for the task of single-user recommendation.

As the proposed approach is based on the group recommendation principles, we performed a comparison of our approach to the standard group recommender (as some researchers argue that group recommender can bring better results as collaborative recommendation) with average aggregation strategy (Figure 3). As we can see, the group recommendation used for single-user brings the worst results. This is an expected result, because of the goal of group recommender -to satisfy whole group. Interesting fact we discovered, is that similar users used for the recommendation construction have no or minimal effect to the precision of generated recommendations (standard group recommendation approach).

Experiments described above on the MovieLens, we performed on the SME.SK dataset respectively. When comparing proposed approach (with various settings as the group size and the number of similar users

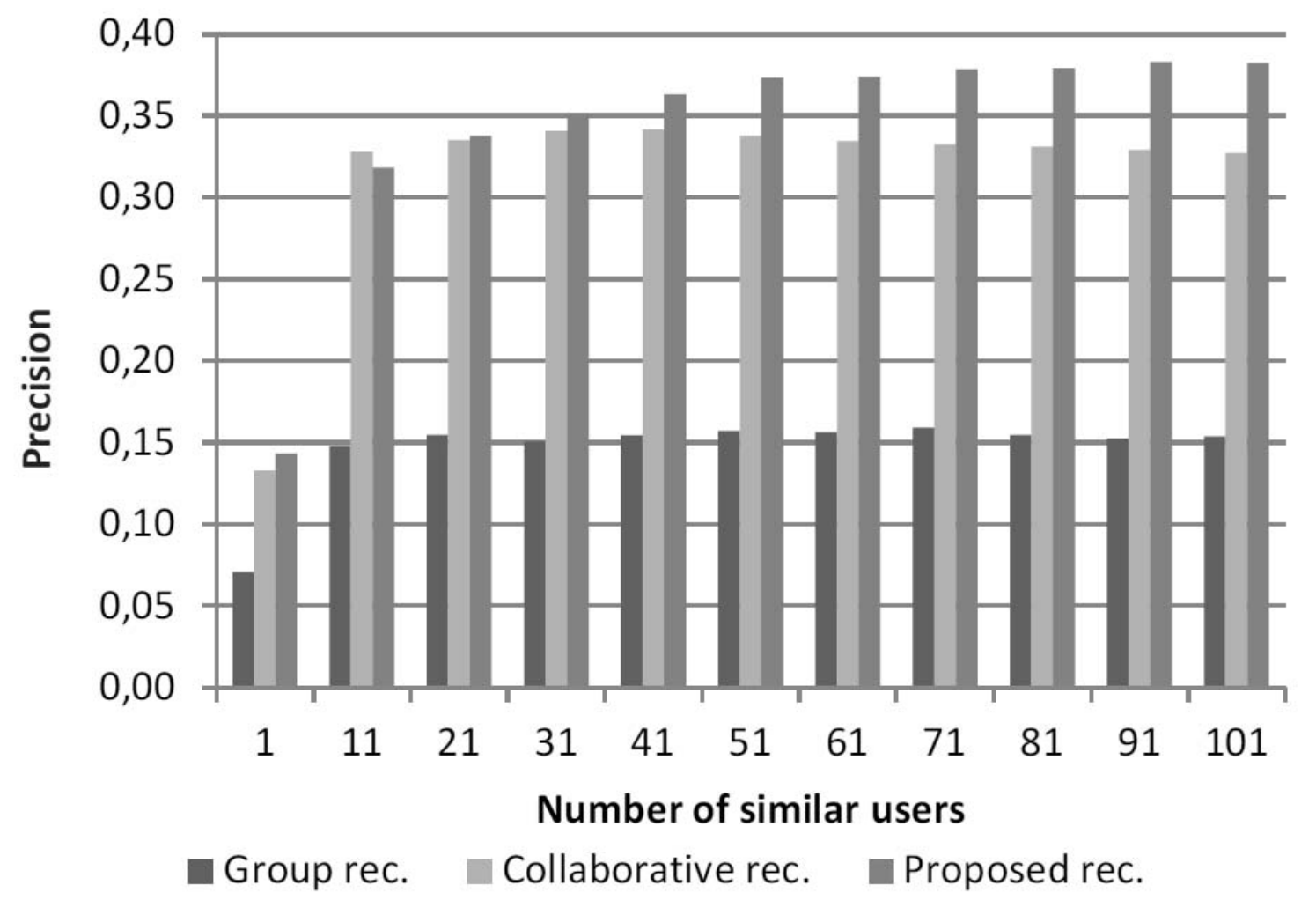

Figure 3. Comparison of the standard group recommender, standard Top-N collaborative approach and proposed single-user group approach for various similar users used for the recommendation (average P@3 for all group sizes). 


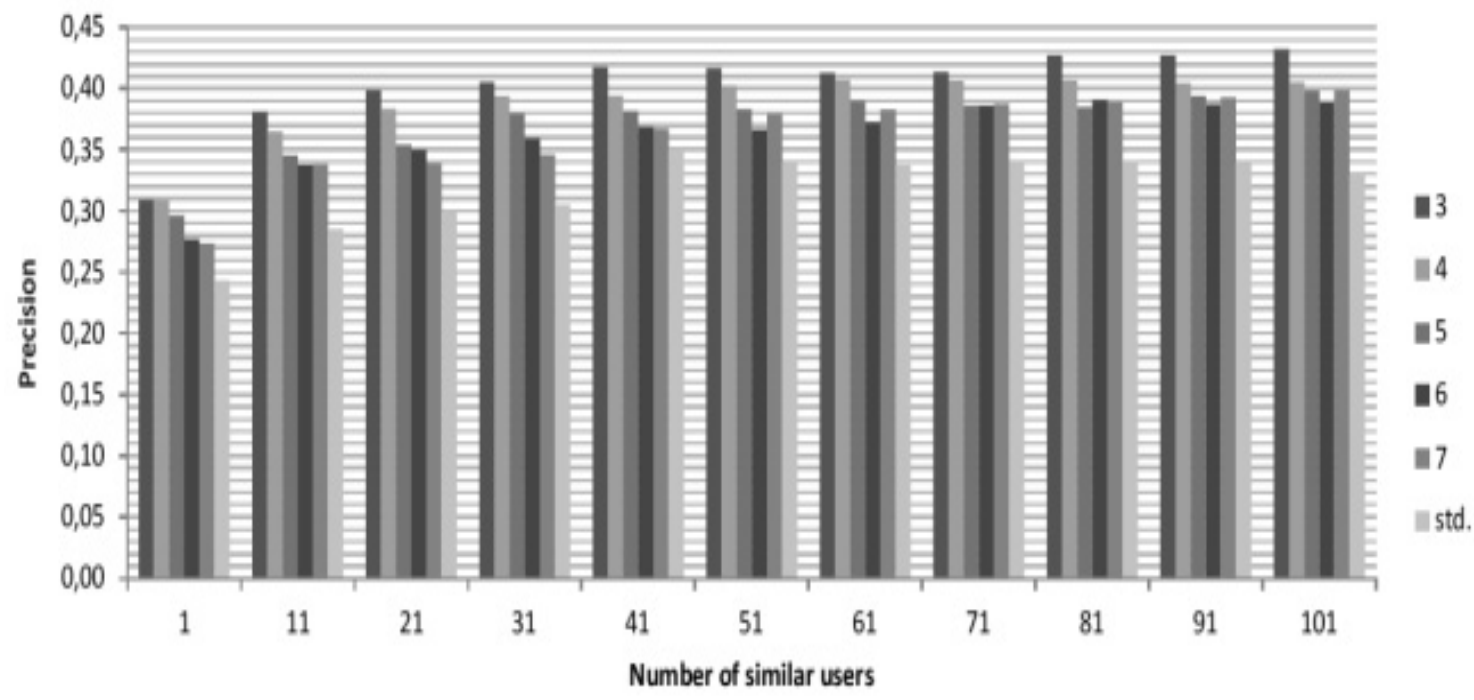

Figure 4. Precision@3 for various numbers of similar users used for the recommendation (1-101) and various group sizes (3-7) - SME.SK dataset. Results of standard collaborative recommender are also presented (std.).

used for recommendation) to the standard single-user collaborative recommender, proposed approach outperforms standard collaborative approach. Similar pattern can be observed over various settings (Figure 4), while the group size of 3 users and 101 similar users respectively, brings the best result (P@3=43.20\%). Proposed group-based approach outperforms the single-user collaborative recommendation in all neighborhoods' sizes (1-101). Because of similar pattern as when used MovieLens dataset, we do not provide detailed results. Similarly, the paired t-test was performed. Obtained results again support our hypothesis that proposed approach improves collaborative recommendation ( $p=0.0001, \alpha=0.05, t=29.10)$. The best improvement over the dataset we observed is $10.01 \%$ (31 users' neighborhood) and the average improvement (SME.SK dataset) over all group and neighborhoods sizes is $8.64 \%$. This brings us to the assumption that our proposed approach can be used for various domains, whose characteristics and users' habits are quite different, while the basic behavior of proposed approach remains unchanged.

\section{Conclusions}

In this paper we proposed new collaborative recommender approach based on the group recommendation principles. The user is assigned to a virtual group created by considering the user to user similarity (comprising not only similar users). Virtual group members' preferences are aggregated in order to create one "virtual" user preference. Finally, these virtual users are used for the collaborative recommendation (virtual users to the real users similarities are computed).

Based on the strategy used for the group construction, other possibilities of proposed approach arise. When combining not active users with some active ones, more recent results can be expected. Similarly, sometimes some kind of influence may be desired teacher of specific class, girlfriend's birthday etc. In such a situation proposed approach consider the preferences of other person and thus recommendations are moved from the single-user preferences. The groups used in proposed approach do not necessarily have to be virtual, but various social aspects can be considered [20].

Highly dynamic domains are not suitable for our approach. In domains where user's preferences change dramatically, the preference aggregation and the most expensive part - the similarity computation have to be performed more often. On the other side, standard collaborative recommenders don't bring sufficient results in domains, where user's preferences or activity is low (not enough similar users to generate recommendation); while proposed approach, due to its virtual user, can provide worthy recommendations. 
Our results of experiments (statistically significant) support our hypothesis that proposed approach overcomes the standard collaborative Top-N recommendation. We compared three aggregation strategies for the group preferences computation. All compared approaches perform very similar and minimal differences were obtained. This brings us to the conclusion that the aggregation strategy is not so important when so small groups are used, as when the standard group recommendation is performed.

Moreover, we discovered that the group size of 3 users overcome other group sizes in the precision, while ratings difference metrics prefer the largest groups of 7 users. This can be explained by the better prediction of possible ratings when more users are involved. Similarly, the inner-group similarity decreases with the group size, because of limited similar user amount. This is in the contrast with standard group recommendation. When comparing to the group recommenders Baltrunas [3] discovered that the group size does not necessarily decreases the performance of group recommendation.

The sizes of the similar users' set, used for the recommendation, influence the results of compared approaches as well. We discovered that for the MovieLens dataset the precision of the standard collaborative approach decreases with the similar user set size increase. Our proposed approach brings the best results when 91 and 101 similar users were used. The statistically significant improvement of our approach was more than 10\% in average (P@3 and P@10). Equally, when SME.SK dataset was used, the average precision improvement approx. 8.64\% was observed. This seems to be a promising result, which support the usage of proposed approach for the single-user recommendation.

As the proposed approach does not consider items' content, various domains (where the content analysis is impossible e.g., music, TV) can be used for the recommendation. Results obtained from different datasets (MovieLens, news portal) support this hypothesis.

\section{Acknowledgements}

This work was supported by grants No.VG1/0675/11, APVV 0208-10 and it is a partial result of the Research and Development Operational Program for the project Support of Center of Excellence for Smart Technologies, Systems and Services, ITMS 26240120005 and ITMS 26240120029 co-funded by ERDF.

\section{REFERENCES}

1. ADOMAVICIUS, G., A. TUZHILIN, Towards the Next Generation of Recommender Systems: A Survey of the State-of-the-art and Possible Extensions, IEEE Transactions on Knowledge and Data Engineering, 2005, pp.734-749.

2. ARDISSONO, L., A. GOY, G. PETRONE, P. SEGNAN, P. TORASSO, Intrigue: Personalized Recommendation of Tourist Attractions for Desktop and Hand held Devices, Applied Artificial Intelligence, 2003, pp. 687-714.

3. BALTRUNAS, L., F. RICCI, Group Recommendations with Rank Aggregation and Collaborative Filtering, In Proceedings of the 4th ACM Conference on Recommender Systems, ACM 2010, pp. 119-126.

4. BORATTO, L., S. CARTA, State-of-theart in Group Recommendation and New Approaches for Automatic Identification of Groups, In Information Retrieval and Mining in Distributed Environments, vol. 324 of Studies in Computer Intelligence, Springer, 2011, pp. 1-20.

5. BRUSILOVSKY, P., A. KOBSA, W NEJDL, The Adaptive Web, In Lecture Notes in Computer Sciences, vol. 4321, Springer, 2007, p. 762.

6. CANDILLIER, L., F. MEYER, M. BOULLÉ, Comparing State-of-the-Art Collaborative Filtering Systems, In Proceedings of the 5th International Conference on Machine Learning and Data Mining in Pattern Recognition, Springer, 2007, pp. 548-562. 
7. CREMONESI, P., Y. KOREN, R. TURRIN, Performance of Recommender Algorithms on Top-n Recommendation Tasks, In Proceedings of the 4th ACM Conference on Recommender Systems, 2010, pp. 39-46.

8. DAS, A. S., M. DATAR, A. GARG, S. RAJARAM, Google News Personalization: Scalable Online Collaborative Filtering, In Proceedings of the 16th International Conference on World Wide Web, ACM 2007, pp. 271-280.

9. GONG, S. J., A Collaborative Recommender Based on User Information and Item Information, In Proceedings of the International Symposium on Information Processes, Academy Publisher, 2009, pp. 1-4.

10. JUNG, H., S. PARK, Pattern Searching in a Social Network, Studies in Informatics and Control, vol. 19(2), 2010, pp. 125-134.

11. KOMPAN, M., M. BIELIKOVÁ, Content-Based News Recommendation, In Lecture Notes in Business Information Processing, vol. 61, Springer, 2010, pp. 61-72.

12. KOMPAN, M., M. BIELIKOVÁ, Group Recommendations: Survey and Perspectives, In Computing and Informatics. 2013. [to appear]

13. KOREN, Y., Advances in Collaborative Filtering, In F. Ricci, L. Rokach, B. Shapira, \& P. B. Kantor (Eds.), Recommender Systems Handbook, Springer, 2011, pp. 145-186.

14. MASTHOFF, J., Group Modelling: Selecting a Sequence of Television Items to Suit a Group of Viewers, In Proceedings of the International Conference on User Modelling and UserAdapted Interaction, Springer, 2004, pp. 37-85.

15. MASTHOFF, J., Group Recommender Systems: Combining Individual Models, In F. Ricci, L. Rokach, B. Shapira, and P. B. Kantor, editors, Recommender Systems Handbook, Springer, 2011, pp. 677-702.
16. MCCARTHY, J. F., T. D. ANAGNOST, MUSIC FX: An Arbiter of Group Preferences for Computer Supported Collaborative Workouts, In Proceedings of the 1998 ACM Conference on Computer Supported Cooperative Work, ACM, 1998, pp. 363-372.

17. MCCARTHY, K., M. SALAMO, L. COYLE, L. MCGINTY, B. SMYTH, P. NIXON, Cats: A Synchronous Approach to Collaborative Group Recommendation, In Proceeding of FLAIRS Conf., AAAI Press, 2006, pp. 86-91.

18. MICHALCO, J., P. NÁVRAT, Arrangement of Face-to-Face Meetings using Social Media, Studies in Informatics and Control, vol. 21(4), 2012, pp. 383-392.

19. NTOUTSI, I., K. STEFANIDIS, K. NORVAG, H. P. KRIEGEL, gRecs: A Group Recommendation System based on User Clustering, In Proceedings of the 17th International Conference on Database Systems for Advanced Applications, Springer, 2012, pp. 1-4.

20. RECIO-GARCIA, J. A., G. JIMENEZDIAZ, A. A. SANCHEZ-RUIZ, B. DIAZAGUDO, Personality Aware Recommendations to Groups, In Proceedings of the 3rd ACM Conference on Recommender Systems, ACM, 2009, pp. 325-328.

21. SENOT, C., D. KOSTADINOV, M. BOUZID, J. PICAULT, A. AGHASARYAN, C. BERNIER, Analysis of Strategies for Building Group Profiles, User Modelling, Adaptation and Personalization, Springer, 2010, pp. 40-51.

22. SHANI, G., A. GUNAWARDANA, Evaluating Recommendation Systems, In F. Ricci, L. Rokach, B. Shapira, and P. B. Kantor, editors, Recommender Systems Handbook, Springer, 2011, pp. 257-297.

23. SU, X., T. M. KHOSHGOFTAAR, A Survey of Collaborative Filtering Techniques, Advances in Artificial Intelligence, 2009, pages 1-20. 
24. SUCHAL, J., P. NÁVRAT, Full Text Search Engine as Scalable k-nearest Neighbour Recommendation System, In Artificial Intelligence in Theory and Practice III, vol. 331 of IFIP Advances in Information and Communication Technologies, Springer, 2010., pp. 165-173.

25. ŠALOUN, P., Z. VELART, Evaluation of Concept Space Rating Algorithm and Navigation Scheme, In Proceedings of the International Conference on Web
Intelligence and Intelligent Agent Tech., vol. 3, 2010, pp. 288-291.

26. WANG, Z., M. ZHANG, Y. TAN, W. WANG, Y. ZHANG, L. CHEN, Recommendation Algorithm Based on Graph Model Considering User Background Information, Proceedings of the 9th International Conference on Creating, Connecting and Collaborating through Computing, 2011, pp. 32-39. 